\title{
Revealing the Origin of "Phonon Glass-Electron Crystal" Behavior in Thermoelectric Layered Cobaltate by Accurate Displacement Measurement
}

\author{
L. Wu ${ }^{1}$, Q. Meng ${ }^{1}$, Ch. Jooss ${ }^{2}$, J.-C. Zheng ${ }^{3}$, H. Inada ${ }^{4}$, D. $\mathrm{Su}^{1}$, Q. Li ${ }^{1}$, and Y. Zhu ${ }^{1}$ \\ ${ }^{1}$ Brookhaven National Laboratory, Upton, NY 11973 USA \\ ${ }^{2}$ University of Goettingen, Goettingen, Germany \\ ${ }^{3}$ Xiamen University, Xiamen, China, \\ ${ }^{4}$ Hitachi High Technologies Corp., Ibaraki Japan
}

Measurement of local disorder and lattice vibrations is of great importance for understanding the mechanisms whereby thermoelectric materials efficiently convert heat to electricity. Calcium cobalt oxides $\left(\mathrm{Ca} 2 \mathrm{CoO}_{3}\right)_{0.62} \mathrm{CoO}_{2}$ is a model system in this regard with a figure of merit $\mathrm{ZT}$ above one. The compound has a complex misfit layered structure with significant lattice displacement (both static and dynamic) that is attributed to the reduced thermal conductivity. Its averaged structure consists of two interpenetrating subsystems of a $\mathrm{CdI}_{2}$-type $\mathrm{CoO}_{2}$ layer and a distorted tri-layered rock-salt-type $\mathrm{Ca}_{2} \mathrm{CoO}_{3}$ block (Fig.1,2), being incommensurately modulated along the b-axis. It is well known that both static displacement and thermal atomic vibration can effectively scatter phonons to reduce thermal conductivity, however, the exact scattering mechanisms are still unknown, largely because there is no reliable method available for such a measurement that can link the displacement to the phonon scattering.

Here, we demonstrate that the quantitative acquisition of multiple annular-dark-field images via STEM at different scattering-angles simultaneously (Fig.1) allows us not only to separate but also accurately determine static and thermal atomic displacement in crystals. This is because the intensity characteristics of a STEM image acquired with high angle annular dark field (HAADF) and medium angle annular dark field (MAADF) differ considerably, depending on the nature of the displacement (Fig.2a-b). Unlike diffraction analysis that derives the overall displacement from the intensities of Bragg reflections, we directly measure the atomic displacement in real space, thereby enabling us to refine independently the atomic displacement in the same lattice planes, i.e., in the rigid $\mathrm{CoO}_{2}$ and soft $\mathrm{Ca}_{2} \mathrm{CoO}_{3}$ layers (Fig.3), that is crucial to revealing their different nature in phonon scattering.

Applying our unique method to the layered thermoelectric material $\left(\mathrm{Ca}_{2} \mathrm{CoO}_{3}\right)_{0.62} \mathrm{CoO}_{2}$ disclosed the presence of large incommensurate displacive modulation and enhanced local vibration of atoms, largely confined within its $\mathrm{Ca}_{2} \mathrm{CoO}_{3}$ sublayers. Relating the refined disorder to ab-initio calculations of scattering rates is a tremendeous challenge. Based on our approximate calculation of scattering rates, we suggest that this well-defined deterministic disorder engenders static displacement-induced scattering and vibrational induced resonance scattering of phonons as the origin of the phonon glass (Fig.4). Concurrently, the crystalline $\mathrm{CoO}_{2}$ sublayers provide pathways for highly conducting electrons and large thermal voltages.

\section{References:}

[1] Wu, L., Meng, Q., Jooss, Ch., Zheng, J.-C., Inada, H., Su, D., Li, Q., and Zhu, Y., Adv. Funct. Mater. 23, 5728-5736 (2013).

[2] Work was supported by the U.S. DOE, Office of Basic Energy Science, Material Science and Engineering Division, under Contract No. DE-AC02-98CH10886. 


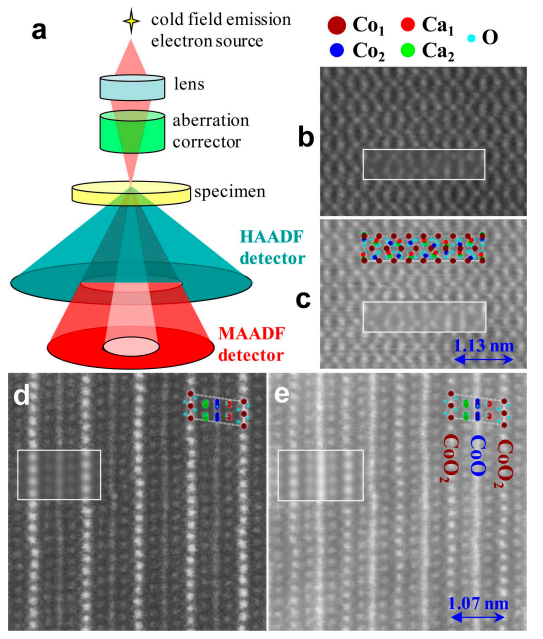

Fig.1 (a) Simultaneous acquisition of HAADF (114-608 mrad, $b, d$ ) and MAADF (46-104 $\operatorname{mrad}, c, e)$ images to determine the static displacive modulation and atomic vibration in the [001] projection $(b, c)$ and the [010] projection $(d, e)$ of $\left(\mathrm{Ca}_{2} \mathrm{CoO}_{3}\right)_{0.62} \mathrm{CoO}_{2}$. The embedded are simulated images.

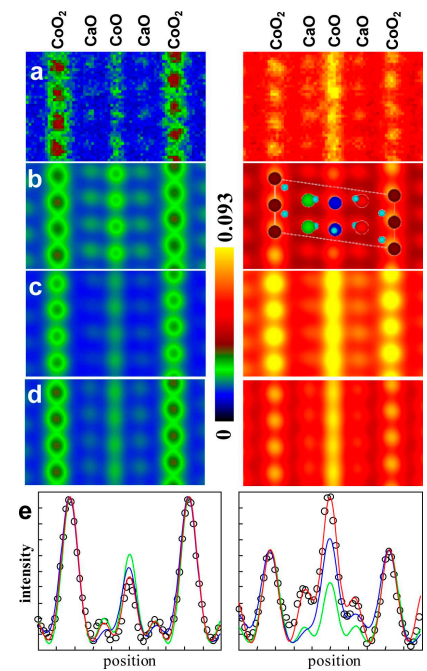

Fig.3 Determining static- \& thermal-displacement in $\left(\mathrm{Ca}_{2} \mathrm{CoO}_{3}\right)_{0.62} \mathrm{CoO}_{2}$. $(a$ to $d)$ simultaneous STEM images in [010] projection. Left panel: HAADF and Right panel: MAADF. (a) Experimental image; Calculated images with Miyazaki's unrelaxed (b), and relaxed model (c) and our refined structure model $(d)$. (e) Profiles by averaging the intensities along the [100] direction. The open circles, and green-, blue-, and red-lines are projected intensity profiles from $(a),(b),(c)$, and $(d)$, respectively. Area thickness is estimated to be $35 \mathrm{~nm}$ from EELS measurement, and finally refined to be $36.3 \mathrm{~nm}$.
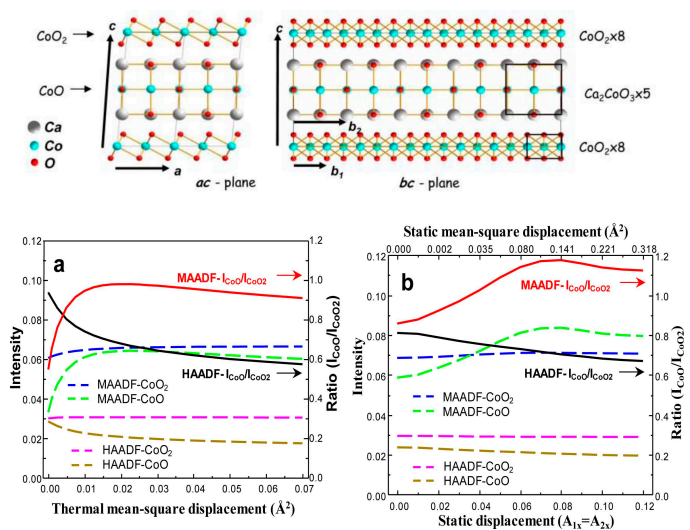

Fig.2 Top: Structural model of $\left(\mathrm{Ca}_{2} \mathrm{CoO}_{3}\right)_{0.62} \mathrm{CoO}_{2}$. Bottom: Image intensity vs displacement. Calculated intensities (dashed lines) in the $\mathrm{CoO}_{2}$ and $\mathrm{CoO}$ layers and intensity ratios $\mathrm{I}_{\mathrm{CoO}} / \mathrm{I}_{\mathrm{CoO} 2}$ (solid lines) for HAADF (collection angle: 114 - 608 mrad) and MAADF (46-104 $\mathrm{mrad})$ as function of $(a)$ thermal mean-square displacement in the CoO layer, and $(b)$ static displacement calculated as two cosine components, $\mathrm{A}_{1 \mathrm{x}}$ and $\mathrm{A}_{2 \mathrm{x}}$, of the Fourier terms of the modulation waves and the equivalent mean-square static displacement in the CoO layer. The image intensities were calculated for $31.7 \mathrm{~nm}$ thickness and averaged over the [100] direction. We note the intensity variation due to thermal- and static-displacements differs significantly in MAADF.
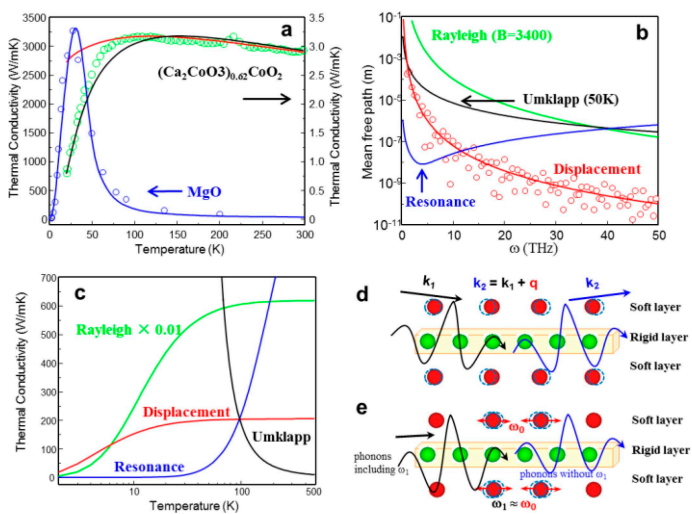

Fig.4 (a) Thermal conductivity vs temperature: circles are experimental data for layered $\left(\mathrm{Ca}_{2} \mathrm{CoO}_{3}\right)_{0.62} \mathrm{CoO}_{2}$ (green, right vertical axis) and cubic $\mathrm{MgO}$ (blue, left vertical axis). Solid lines are fitting curves based on specific heat, DOS, group velocity, and Mean free path (MFP). The blue and red lines are fitting only containing the Umklapp and Rayleigh terms, while the black line including the displacement and resonance terms. (b) MFP for Umklapp scattering, Rayleigh scattering, modulation-induced-displacement and resonance scattering. (c) Individual contributions from different scattering mechanisms on thermal conductivity as a function of temperature. $(d-e)$ Schematics of displacement scattering $(d)$ and resonance scattering $(e)$. Atoms in the soft layer deviate from its periodical positions in $(d)$ due to the displacement, resulting in phonon scattering. (e) Phonons with frequency of $\omega_{1}$ tune into resonance with a local vibration at $\omega_{0}\left(\omega_{1} \approx \omega_{0}\right)$ will be absorbed, those outside of the resonance frequency band will propagate. 